\title{
Afferent Loop Syndrome With Associated Perforation 15 Years After Gastrectomy And Roux-En-Y Reconstruction
}

\section{Abstract}

An elderly patient presented to our institution with abdominal pain, nausea and vomiting. She had an extensive surgical background including gastrectomy with Roux-en-y reconstruction undertaken 15 years earlier. A CT abdomen demonstrated a small bowel obstruction proximal to the jejunojejunostomy with features suggestive of afferent loop syndrome (ALS), a rare but known complication following Roux-en-y reconstruction. Initial surgery identified a band adhesion and an associated ischemic small bowel segment, which was resected with primary anastomosis. A further operation was required at post-operative day five due to clinical deterioration. A retroperitoneal collection was identified secondary to perforation of the afferent loop.

\section{Background}

This is a rare case and potentially the first case report in the literature of intestinal perforation secondary to afferent loop syndrome. Afferent loop syndrome is a life threatening condition with mortality reported at 30-60\% in the literature. As such, in a patient presenting with obstructive symptoms with a background of gastrectomy with Roux-en-y reconstruction it is important to consider ALS as a potential diagnosis and prompt surgical management is required.

\section{Case presentation}

Mrs M, a 71 year old female presented to our institution following six days of increasing epigastric pain with associated nausea and vomiting. Her bowels had not opened for five days prior to admission. She denied febrile symptoms.

Mrs $\mathrm{M}$ has a significant surgical background with a vagotomy and distal gastrectomy for peptic ulcer disease in 1999, completion gas- 
trectomy with Roux-en-Y reconstruction that same year, incisional hernia repair with mesh 2002, an open cholecystectomy for acute cholecystitis in 2005 and a previous open appendicectomy.

She is on a multitude of medications including opioids for osteoarthritis and levothyroxine for hypothyroidism. She has a history of alcoholism.

On examination she was normothermic, was noted to have new atrial fibrillation (AF) with a rate of 170 beats per minute (bpm) and was hypotensive at $92 / 58 \mathrm{mmHg}$. She was not in respiratory distress.

Her abdomen was soft with tenderness across the upper abdomen, most severe in the epigastrium. There were no signs of peritonism and bowel sounds were scant.

\section{Investigations}

Initial blood tests revealed a mild leukocytosis and C-reactive protein of $546 \mathrm{mg} / \mathrm{L}$. Haemoglobin and renal function were normal with mildly deranged liver function tests with the bilirubin 26 umol/L (220), ALP 272 U/L (30-150), ALT 44 U/L (5-30). The amylase was normal.

A CT abdomen /pelvis demonstrated a small bowel obstruction of the enteric limb with the transition point just proximal to the jejunojejunostomy. There was a retroperitoneal air and fluid filled collection noted in the right upper quadrant but was thought to be intraluminal. Furthermore, marked biliary ductal dilatation was noted which had increased significantly since her previous CT Abdomen in 2012.

\section{Differential diagnosis}

Given the clinical presentation and CT findings the diagnosis of afferent loop syndrome was made.

\section{Treatment}

Mrs $M$ was taken acutely to theatre on the day of presentation. A laparotomy was undertaken which demonstrated a band adhesion causing an ischaemic segment of small bowel with proximal dilatation of the biliary limb (afferent limb), with gross distension of the distal duodenum. There was minimal free fluid. There were marked adhesions in the right upper quadrant and as there did not appear to be any pathology there intraoperatively this was not explored. The distal small bowel was collapsed, as was the colon. A limited small bowel resection with primary anastomosis was undertaken. Histology of the small bowel segment confirmed haemorrhagic necrosis consistent with ischemia.

Post-operatively she was transferred to the intensive care unit where parenteral nutrition was commenced. Intravenous amiodarone was started for her atrial fibrillation. She progressed well with satisfactory post-operative laboratory tests and was transferred to the ward with continued input from the internal medicine and cardiology teams.

She had minimal output from her nasogastric tube and was commenced on clear fluids on postoperative day four.

On day five she reported increasing upper abdominal pain and distension. Although her observations remained stable abdominal examination revealed signs of peritonism in the right upper quadrant. Laboratory tests demonstrated an increasing white cell count of 20 109/L (4-11) and marked derangement in her liver function tests (bilirubin 44, ALP 828, ALT 120, GGT 2529, AST 156).

A repeat $C T$ abdomen suggested only a small amount of free air consistent with previous surgery with no significant fluid collection adjacent to the anastomosis from the recent surgery. The possible collection mentioned in the previous scan in the right upper quadrant was still present and of similar size and was again thought to be intraluminal. Given her rising inflammatory markers broad spectrum antibiotics were started.

Due to the clinical picture with failure to progress, it was decided to proceed with a further laparotomy. The anastomosis was intact with no sign of a leak 
or other pathology. The right upper quadrant was further explored with adhesions taken down. There was no obvious abnormalities initially however further dissection laterally was carried out to refute or confirm the CT finding of a large intraluminal air and fluid collection. There was a large collection of pus and enteric content in the retroperitoneal space. On closer inspection it was a perforation of the biliary limb. The collection was washed out and two large drains were placed and a feeding jejunostomy created. She was commenced on octreotide.

\section{Outcome and follow-up}

By post-operative day five from her second operation her drain output had ceased and a repeat CT scan showed near complete resolution of the collection and a marked reduction in the intrahepatic biliary dilatation.

She was discharged home four weeks later after spending time in rehabilitation ward.

\section{Discussion}

Retrospective analysis of the CT examination led to the conclusion that the perforation occurred prior to admission and in a diverticulum in the afferent limb (Figure 1). This was deemed secondary to the closed loop obstruction that had developed. The first and second CT scans had revealed the same collection but had been misreported. Due to marked adhesions and the location of the collection in the retroperitoneum this was not identified at the time of the initial operation. She improved initially after the first laparotomy secondary to firstly resecting the ischaemic segment but secondly the collection would have been temporarily decompressed at the time the bowel was opened for the small bowel anastomosis.

Afferent loop syndrome (ALS), also termed afferent limb/biliopancreatic limb syndrome, is a known but rare complication following gastrectomy with Roux-en-y reconstruction. ALS and its association with gastric surgery were first described in the lite-
Figure 1: Axial CT abdomen demonstrating RUQ collection.

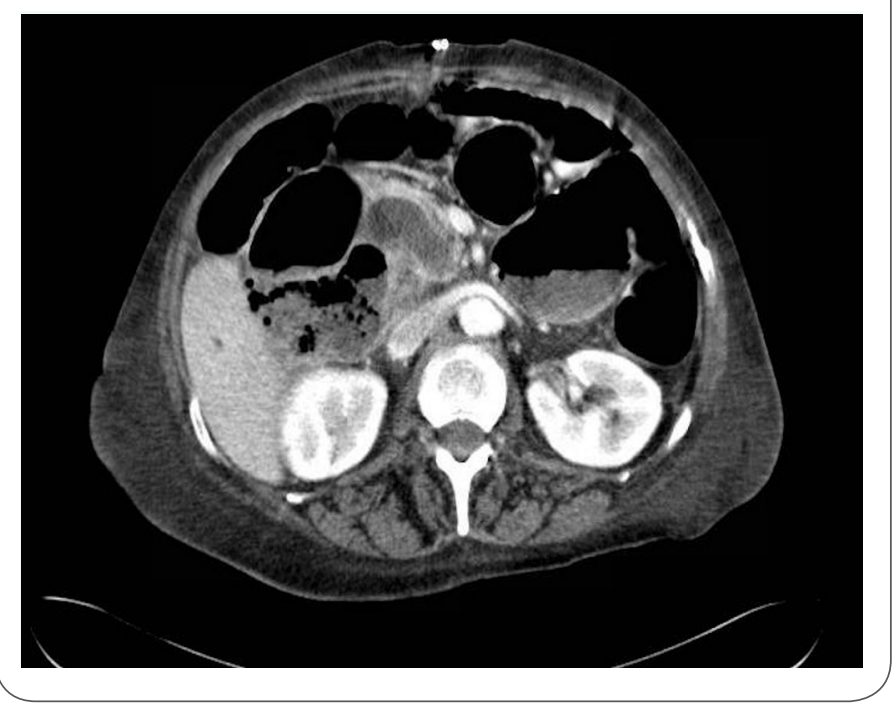

rature in 1881 and since then advances in surgical techniques have reduced the incidence of the condition in the post-operative period [1].

ALS most often occurs in the weeks to months following the initial surgery however rare cases have been known to present many years after surgery. One case report illustrates a patient who developed ALS 27 years following a subtotal gastrectomy with Roux-en-y reconstruction [2].

Obstruction of the small bowel downstream from the afferent loop and subsequent dilation of the afferent loop can occur as a result of adhesions, internal herniation, volvulus, intussusception, stenosis from malignancy, inflammation or large stones [3]. A retrospective review of 1908 patients following gastrectomy with Roux-en-y reconstruction found that four patients (0.2\%) went on to develop ALS, the causes being internal herniation in two, adhesion in one and peritoneal recurrence in the other. The period between initial and emergency operations had a median of 5 months [4].

Presenting symptoms are usually abdominal pain, nausea and vomiting and depending on the severity of the underlying pathology may have signs of peritonism on examination. As in our case deranged liver function tests and an elevated amylase with 
signs of pancreatitis may result from fluid build up in the afferent limb and associated dilation of the biliary and pancreatic ducts [5].

Abdominal x-ray is usually unhelpful in the diagnosis as the afferent limb is a fluid-filled structure. $\mathrm{CT}$ is the most reliable modality in diagnosis and findings suggestive of ALS often include a dilated afferent limb, which is usually situated in the midabdomen crossing between the aorta and mesenteric vessels. Biliary and pancreatic duct dilatation is a common finding [2].

ALS is a serious condition with an estimated mortality rate of $30-60 \%$ and patients require urgent surgical management to relieve the underling cause [4].

With progressive dilation of the afferent loop perforation is an obvious potential complication of ALS however following review of the literature we were unable to a find a case report illustrating perforation in ALS.

Although a rare complication, our case illustrates that ALS should always be considered in a patient with a history of gastrectomy with Roux-en-y reconstruction presenting with abdominal pain and symptoms/signs of obstruction. It is a potentially life threatening complication which can result in perforation of the afferent loop and urgent surgery to correct the underlying pathology should be undertaken. The option of a less invasive radiologically guided percutaneous drain could also be entertained in the specific clinical situation and may have been appropriate as a substitute for the second laparotomy. This was not done in our case as the diagnosis of perforation was made intraoperatively. This case may be the first in the literature describing a perforation of the afferent limb in ALS.

\section{Learning points/take home messages}

- Always consider ALS in patients presenting with obstructive symptoms on a background of Roux-en-y reconstruction.

- The syndrome often involves deranged LFTs and pancreatic enzymes.
- Urgent surgical management to treat the underlying condition is required.

- Perforation of the afferent limb is a rare but potential complication in ALS.

\section{References}

1. Mitty WF, Grossi C, Nealon T. Chronic Afferent Loop Syndrome. Annals of Surgery. 1970; 172: 996-1001.

2. Ballas KD, Rafailidis SF, Konstantinidis HD, Pavlidis TE, Marakis GN, Anagnostara E et al. Acute afferent loop syndrome: A true emergency. A case report. Acta Chir Belg. 2009; 109: 101-103.

3. Gayer G, Barsuk D, Hertz M, Apter S, Zissin R. CT Diagnosis of Afferent Loop Syndrome. Clinical Radiology. 2002; 57: 835-839.

4. Aoki M, Saka M, Morita S, Fukagawa T, Katai H. Afferent loop obstruction after distal gastrectomy with Roux-en-Y reconstruction. World J Surg. 2010; 34: 2389-2392.

5. Beveridge CJ, Zammit-Maempel I. Afferent loop obstruction CT appearances of an unusual cause of acute pancreatitis. Clin Radiology. 1999; 54: 188-9.

\section{Comment on this article:}

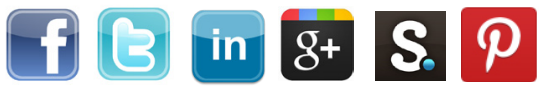

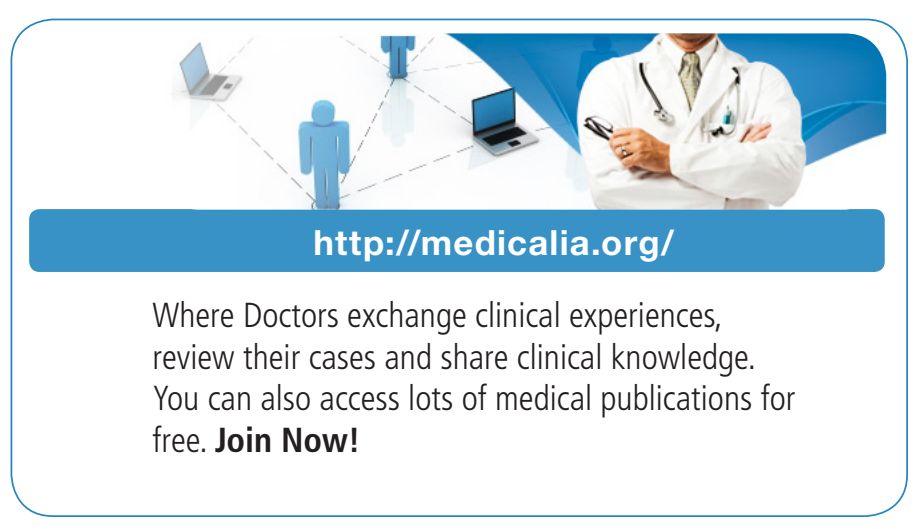

\section{Publish with iMedPub}

\section{http://www.imed.pub}

International Archives of Medicine is an open access journal publishing articles encompassing all aspects of medical science and clinical practice. IAM is considered a megajournal with independent sections on all areas of medicine. IAM is a really international journal with authors and board members from all around the world. The journal is widely indexed and classified Q1 in category Medicine. 